Pacific Journal of Mathematics

EXPONENTIAL REPRESENTATION OF SOLUTIONS TO AN
ABSTRACT SEMI-LINEAR DIFFERENTIAL EQUATION 


\title{
EXPONENTIAL REPRESENTATION OF SOLUTIONS TO AN ABSTRACT SEMI-LINEAR DIFFERENTIAL EQUATION
}

\author{
G. F. WEBB
}

It is shown that the solutions to the abstract differential equation $u^{\prime}=-(A+B) u, u(0)=x \in X$, where $X$ is a Banach space, $-A$ is a linear analytic semigroup generator, and $B$ is Lipschitz continuous from the domain of a fractional power of $A$ to $X$, have the exponential representation $u(t)=$ $\lim _{n \rightarrow \infty}(I+t / n(A+B))^{-n} x$.

1. Introduction. Let $X$ be a Banach space with norm \|\| . We are concerned with the abstract semi-linear differential equation in $X$

$$
d u(t) / d t=-(A+B) u(t), \quad t>0, \quad u(0)=x \in X
$$

where $-A$ is the generator of an analytic semigroup of linear operators in $X$ and $B$ is Lipschitz continuous from the domain of a fractional power of $A$ to $X$. The objective of this paper is to obtain the exponential representation of the solutions to (1.1) in the form

$$
u(t)=\lim _{n \rightarrow \infty}(I+t / n(A+B))^{-n} x .
$$

Exponential representations of the form (1.2) are very well known for the case that $A$ and $B$ satisfy accretive type conditions (see, e.g., [1] and [8]). In the accretive case the nonlinear resolvent $(I+t / n(A+B))^{-1}$ is Lipschitz continuous with

$$
\left|(I+t / n(A+B))^{-1}\right|_{\text {Lip }} \leqq(1-t \gamma / n)^{-1}, t \geqq 0, n \text { sufficiently large, }
$$

where $\gamma$ is some real constant. In our case the main difficulty in establishing (1.2) is that the nonlinear resolvent satisfies a more general condition of the form

$$
\left|(I+t / n(A+B))^{-n}\right|_{\text {LIP }} \leqq M(1-t \gamma / n)^{-n}, t \geqq 0, n \text { sufficiently large, }
$$

where $M$ and $\gamma$ are real constants and $M>1$.

We make the following assumption on $A$ : 
(1.3) there exists $\omega<0$ such that $A=A_{0}-\omega I$, where $A_{0}$ is a closed densely defined linear operator from $X$ to $X$, the resolvent set of $A_{0}$ contains the sector $S_{\Phi}=\{\lambda: \lambda \neq 0, \pi / 2-\Phi<\arg \lambda<$ $3 \pi / 2+\Phi\}$, $\Phi$ some constant in $(0, \pi / 2)$, and $\left|\left(\lambda I-A_{0}\right)^{-1}\right| \leqq M /|\lambda|$ for $\lambda \in S_{\Phi}$, where $M$ is a given constant $\geqq 1$.

As a consequence of (1.3) we have (see [3]):

(1.4) $-A$ is the infinitesimal generator of an analytic semigroup of linear operators $T(t), t \geqq 0$ in $X,|T(t)| \leqq M e^{\omega t}$ for $t \geqq 0$, and $\left|(I+t / n A)^{-n}\right| \leqq M(1-\omega t / n)^{-n}$ for $t \geqq 0$ and $n$ a positive integer;

(1.5) if $0<\alpha<1$ then the fractional power $A^{-\alpha}$ is defined as a bounded linear operator in $X$ by $A^{-\alpha}=(1 / \Gamma(\alpha)) \int_{0}^{\infty} e^{-s A} s^{\alpha-1} d s$, and $D\left(A^{\alpha}\right)$ is a Banach space with norm $\|x\|_{\alpha} \stackrel{\text { def }}{=}\left\|A^{\alpha} x\right\|$ for $x \in D\left(A^{\alpha}\right)$;

(1.6) if $0<\alpha<1$, then there exists $C>0$ such that $\left|A^{\alpha} T(t)\right| \leqq C e^{\omega t} t^{-\alpha}$ for $t>0$.

We make the following assumption on $B$ :

(1.7) there exists $\alpha \in(0,1)$ such that $B$ is an everywhere defined operator from $D\left(A^{\alpha}\right)$ to $X$ and there exists $L>0$ such that $\|B x-B y\| \leqq L\|x-y\|_{\alpha}$ for $x, y \in D\left(A^{\alpha}\right)$.

Under the assumptions (1.3) and (1.7) and the assumption that $x \in D\left(A^{\alpha}\right)$ the equation (1.1) can be integrated to yield the equivalent integral equation

$$
u(t)=T(t) A^{\alpha} x-\int_{0}^{t} A^{\alpha} T(t-s) B A^{-\alpha} u(s) d s, \quad t \geqq 0 .
$$

(see, e.g., [5], Chapter 3). The equation (1.8) is a singular Volterra integral equation and has been subject of extensive study. The case in which $B: D\left(A^{\alpha}\right) \rightarrow X$ is locally Lipschitz continuous is treated in [5], the case in which $A^{-1}$ is compact and $B: D\left(A^{\alpha}\right) \rightarrow X$ is Holder continuous is treated in [3], and the case in which $B$ is accretive and $B: D\left(A^{\alpha}\right) \rightarrow X$ is locally continuous is treated in [7]. In our treatment of (1.8) the following lemma (which is similar to Gromwall's lemma) will play a fundamental role (see also [3], Theorem 7.1.1):

Lemma 1.1. Let $w:\left[0, t_{0}\right] \rightarrow[0, \infty)$ be continuous, let $a \geqq 0, b \geqq 0$, 
$\omega \in R, 0<\alpha<1$, and let

$$
w(t) \leqq a e^{\omega t}+b \int_{0}^{t} e^{\omega(t-s)}(t-s)^{-\alpha} w(s) d s, \quad 0 \leqq t \leqq t_{0} .
$$

Then, for all real $\gamma$ such that $\gamma>\omega$ and $b \Gamma(1-\alpha)(\gamma-\omega)^{\alpha-1}<1$, we have that

$$
w(t) \leqq a\left(1-b \Gamma(1-\alpha)(\gamma-\omega)^{\alpha-1}\right)^{-1} e^{\gamma t}, \quad 0 \leqq t \leqq t_{0} .
$$

Proof. We will use the gamma function formula

$$
\Gamma(z)=\beta^{z} \int_{0}^{\infty} e^{-\beta s} s^{z-1} d s \quad \text { for } \quad z>0, \quad \beta>0
$$

(see [9], p. 265). Let $\gamma$ be as above, and let $S=\sup _{0 \leqq t \leqq t_{0}} e^{-\gamma t} w(t)$. For $0 \leqq t \leqq t_{0}(1.9)$ implies

$$
\begin{aligned}
e^{-\gamma t} w(t) & \leqq a e^{(\omega-\gamma) t}+b \int_{0}^{t} e^{(\omega-\gamma)(t-s)}(t-s)^{-\alpha} e^{-\gamma s} w(s) d s \\
& \leqq a+b S \Gamma(1-\alpha)(\gamma-\omega)^{\alpha-1}
\end{aligned}
$$

Thus, $S \leqq a+b S \Gamma(1-\alpha)(\gamma-\omega)^{\alpha-1}$, which implies (1.10).

2. Existence of solutions. It is well known that under the assumptions (1.3) and (1.7) there exixts a solution to (1.8) for each $x \in D\left(A^{\alpha}\right)$ (see, e.g., [5], Theorem 3.3.3). We will prove this fact below, however, since our proof will be instructive for the techniques we use to prove (1.2).

Proposition 2.1. Let (1.3) and (1.7) hold and let $x \in$ $D\left(A^{\alpha}\right)$. There exists a unique continuous function $u:[0, \infty) \rightarrow D\left(A^{\alpha}\right)$ satisfying

$$
u(t)=T(t) x-\int_{0}^{t} T(t-s) B u(s) d s, \quad t \geqq 0 .
$$

Proof. Let $\gamma$ be real such that

$$
\gamma>\omega \text { and } \operatorname{CL} \Gamma(1-\alpha)(\gamma-\omega)^{\alpha-1}<1
$$

and let $t_{0}>0$. Let $Y$ be the Banach space of all continuous functions $u$ from $\left[0, t_{0}\right]$ to $X$ with norm $\|u\|_{Y}=\sup _{0 \leqq t \leqq t_{0}} e^{-\gamma t}\|u(t)\|$. Define the mapping $F: Y \rightarrow Y$ by 


$$
(F u)(t)=T(t) A^{\alpha} x-\int_{0}^{t} A^{\alpha} T(t-s) B A^{-\alpha} u(s) d s, \quad 0 \leqq t \leqq t_{0} .
$$

We will use (1.11) to show that $F$ maps $Y$ into $Y$ and $F$ is a contraction on $Y$.

First, we see that $(F u)(t)$ is continuous in $t$ for each $u \in Y$, since for $0 \leqq t_{1} \leqq t_{2} \leqq t_{0}$

$$
\begin{aligned}
&\left\|(F u)\left(t_{1}\right)-(F u)\left(t_{2}\right)\right\| \leqq \\
&+\left\|\int_{t_{1}}^{t_{2}} A^{\alpha} T(s) B A^{-\alpha} u\left(t_{2}-s\right) d s\right\| \\
&+\left\|\left(T\left(t_{1}\right)-T\left(t_{2}\right)\right) A^{\alpha} x\right\| \\
& \leqq \int_{t_{1}}^{t_{2}} C e^{\omega s} s^{-\alpha} \sup _{t_{1} \leqq s \leqq t_{2}} \cdot\left\|B A^{-\alpha} u\left(t_{2}-s\right)\right\| d s \\
&+\int_{0}^{t_{1}} C L e^{\omega s} s^{-\alpha}\left\|u\left(t_{1}-s\right)-u\left(t_{2}-s\right)\right\| d s+\left\|\left(T\left(t_{1}\right)-T\left(t_{2}\right)\right) A^{\alpha} x\right\| .
\end{aligned}
$$

Next, we see that $F$ is a contraction, since for $u, v \in Y, 0 \leqq t \leqq t_{0}$

$$
\begin{aligned}
& e^{-\gamma t}\|(F u)(t)-(F v)(t)\| \\
& \quad \leqq C L \int_{0}^{t} e^{(\omega-\gamma)(t-s)}(t-s)^{-\alpha} e^{-\gamma s}\|u(s)-v(s)\| d s \\
& \quad \leqq\left(C L \Gamma(1-\alpha)(\gamma-\omega)^{\alpha-1}\right)\|u-v\|_{Y}
\end{aligned}
$$

By the contraction mapping theorem there exists a unique $u_{0} \in Y$ such that $F u_{0}=u_{0}$. Define $u(t)=A^{-\alpha} u_{0}(t)$ for $0 \leqq t \leqq t_{0}$ and obviously $u(t)$ satisfies (2.1) uniquely. Furthermore, $u(t) \in D\left(A^{\alpha}\right)$ for $0 \leqq t \leqq t_{0}$ and $u(t)$ is continuous from $\left[0, t_{0}\right]$ to $D\left(A^{\alpha}\right)$ (here we have used the continuity of $\left.A^{-\alpha}\right)$. Since $t_{0}$ is arbitrarily large, the proof is finished.

Definition 2.1. Define the family of operators $U(t), t \geqq 0$ in $D\left(A^{\alpha}\right)$ by $U(t) x=u(t)$, where $u$ is the unique solution of $(2.1)$ for a given $x \in D\left(A^{\alpha}\right)$. Then, $U(t), t \geqq 0$ is a strongly continuous semigroup of nonlinear operators in $D\left(A^{\alpha}\right)$. In fact, we have the following:

Proposition 2.2. Let (1.3) and (1.7) hold and for each $t \geqq 0$ let $U(t): D\left(A^{\alpha}\right) \rightarrow D\left(A^{\alpha}\right)$ be defined as above. Then, $U(t), t \geqq 0$ satisfies the following:

(2.3) $U(0)=I$ and $U(t) X:[0, \infty) \rightarrow D\left(A^{\alpha}\right)$ is continuous in $t$ for each fixed $x \in D\left(A^{\alpha}\right)$; 


$$
U\left(t_{1}+t_{2}\right) x=U\left(t_{1}\right) U\left(t_{2}\right) x \text { for } t_{1}, t_{2} \geqq 0, x \in D\left(A^{\alpha}\right) ;
$$

$\|U(t) x-U(t) y\|_{\alpha} \leqq M\left(1-C L \Gamma(1-\alpha)(\gamma-\omega)^{\alpha-1}\right)^{-1} e^{\gamma t}\|x-y\|_{\alpha}$ for $t \geqq 0, x, y \in D\left(A^{\alpha}\right)$, and $\gamma$ satisfying (2.2).

Proof. (2.3) follows immediately from Proposition 2.1. (2.4) follows from the uniqueness of solutions to (2.1) (see [7], Proposition 3.6). (2.5) follows immediately from Lemma 1.1, since

$$
\begin{aligned}
& e^{-\gamma t}\|U(t) x-U(t) y\|_{\alpha} \leqq M e^{(\omega-\gamma) t}\|x-y\|_{\alpha} \\
& \quad+\int_{0}^{t} C e^{(\omega-\gamma)(t-s)}(t-s)^{-\alpha} L e^{-\gamma s}\|U(s) x-U(s) y\|_{\alpha} d s .
\end{aligned}
$$

Corollary 2.1. If $L<(-\omega)^{1-\alpha} / C \Gamma(1-\alpha)$, then there exists a unique $x_{0} \in D\left(A^{\alpha}\right)$ such that $\lim _{t \rightarrow \infty}\left\|U(t) x-x_{0}\right\|_{\alpha}=0$ for all $x \in D\left(A^{\alpha}\right)$.

Proof. Choose $\gamma<0$ satisfying (2.2) and by (2.5) we have that $U(t)$ is a strict contradiction for $t$ sufficiently large, say $t \geqq t_{1}$. Let $x_{t}$ be the unique fixed point of $U(t)$ for each $t \geqq t_{1}$. If $s, t \geqq t_{1}$, then $U(t) x_{s}=$ $U(t) U(s) x_{s}=U(t+s) x_{s}=U(s) U(t) x_{s}$, which implies $x_{s}=U(t) x_{s}$, and which in turn implies $x_{s}=x_{t}$. The conclusion follows immediately using (2.5).

Definition 2.2. Define the infinitesimal generator of $U(t), t \geqq 0$ to be the nonlinear operator $F: D\left(A^{\alpha}\right) \rightarrow D\left(A^{\alpha}\right)$ given by

$$
D(F)=\left\{x \in D\left(A^{\alpha}\right): \lim _{t \rightarrow 0}(U(t) x-x) / t \text { exists in } D\left(A^{\alpha}\right)\right\}
$$

$F x=\lim _{t \rightarrow 0}(U(t) x-x) / t$ (where the limit is taken in $\left.D\left(A^{\alpha}\right)\right)$.

Proposition 2.3. $D(F)=\left\{x \in D(A):(A+B) x \in D\left(A^{\alpha}\right)\right\} \quad$ and $F x=-(A+B) x$ for all $x \in D(F)$.

Proof. First, let $x \in D(F)$. Then, $X-\lim _{t \rightarrow 0}(U(t) x-x) / t=F x$, since the $\alpha$-norm dominates the $X$-norm. Since $X$ $\lim _{t \rightarrow 0} \int_{0}^{t} T(t-s) B A^{-\alpha} A^{\alpha} U(s) x d s / t=B x \quad$ (for any $\left.x \in D\left(A^{\alpha}\right)\right)$ and since $(T(t) x-x) / t=(U(t) x-x) / t+\int_{0}^{t} T(t-s) B U(s) x d s / t$, we see that $x \in D(A) \quad$ and $\quad X-\lim _{t \rightarrow 0}(U(t) x-x) / t=-(A+B) x$. But then $\alpha-\lim _{t \rightarrow 0}(U(t) x-x) / t=-(A+B) x$ as well, and therefore $(A+B) x \in D\left(A^{\alpha}\right)$ and $F x=-(A+B) x$. 
Now let $x \in D(A)$ and let $(A+B) x \in D\left(A^{\alpha}\right)$. Since $\left(A^{\alpha}+A^{\alpha-1} B\right) x=A^{-1} A^{\alpha}(A+B) x$, we have that

(2.6) $\left(A^{\alpha}+A^{\alpha-1} B\right) x \in D(A)$ and $A\left(A^{\alpha}+A^{\alpha-1} B\right) x=A^{\alpha}(A+B) x$.

Since $(T(t)-I) A^{-1} B x=-\int_{0}^{t} T(s) B x d s$ (see p. 481, [6]), we have that

(2.7) $\quad(T(t)-I) A^{\alpha-1} B x=A^{\alpha}(T(t)-I) A^{-1} B x=-\int_{0}^{t} A^{\alpha} T(s) B x d s$.

Next, we show

(2.8) there is a constant $C_{x}$ depending only on $x$ such that $\|U(t) x-x\|_{\alpha} \leqq t C_{x}$ for $0 \leqq t \leqq 1$.

To prove (2.8) let $0<t_{0} \leqq 1$ and define $w:\left[0, t_{0}\right] \rightarrow[0, \infty)$ by $w(t)=$ $\|U(t) x-x\|_{\alpha}, 0 \leqq t \leqq t_{0}$. Using (2.6) and (2.7) we have for $0 \leqq t \leqq t_{0}$

$$
\begin{aligned}
w(t)= & \|(T(t)-I)\left(A^{\alpha}+A^{\alpha-1} B\right) x-(T(t)-I) A^{\alpha-1} B x \\
& \quad-\int_{0}^{t} A^{\alpha} T(t-s) B U(s) x d s \| \\
= & \left\|\int_{0}^{t} T(s) A\left(A^{\alpha}+A^{\alpha-1} B\right) x+\int_{0}^{t} A^{\alpha} T(t-s)[B x-B U(s) x] d s\right\| \\
\leqq & M t_{0} e^{|\omega| t}\left\|A^{\alpha}(A+B) x\right\|+\int_{0}^{t} C e^{|\omega|(t-s)}(t-s)^{-\alpha} L w(s) d s .
\end{aligned}
$$

Then, (2.8) follows from Lemma 1.1 with $a=M t_{0}\|(A+B) x\|_{\alpha}, b=C L$, and $\omega=|\omega|$. To complete the proof it remains to show that

$$
\lim _{t \rightarrow 0}\|(U(t) x-x) / t+(A+B) x\|_{\alpha}=0 .
$$

For $0<t \leqq 1$

$$
\begin{gathered}
\left\|(T(t) x-x) / t-\int_{0}^{t} T(t-s) B U(s) x d s / t+(A+B) x\right\|_{\alpha} \\
=\|((T(t)-I) / t)\left(A^{\alpha}+A^{\alpha-1} B\right) x+A^{\alpha}(A+B) x \\
\quad+\int_{0}^{t} A^{\alpha} T(t-s)[B x-B U(s) x] d s / t \| .
\end{gathered}
$$

Then, (2.9) follows using (2.6), (2.8), and the estimate 


$$
\begin{aligned}
& \left\|\int_{0}^{t} A^{\alpha} T(t-s)[B x-B U(s) x] d s / t\right\| \\
& \quad \leqq C L \int_{0}^{t} e^{\omega(t-s)}(t-s)^{-\alpha} C_{x} d s \\
& \quad \leqq \text { const } t^{1-\alpha} .
\end{aligned}
$$

3. Exponential representation of solutions. Before proving (1.2) we require the following lemmas:

Lemma 3.3. Let $0<\alpha<1$. There exists a constant $K$ such that if $\beta>0, t>0$, and $n$ is a positive integer, then

$$
(t / n)^{1-\alpha} \sum_{k=1}^{n} \Gamma(k-\alpha) e^{-t \beta k / n} / \Gamma(k)<K \Gamma(1-\alpha) \beta^{\alpha-1} .
$$

Proof. We will use the fact that there exists a constant $K$ such that

$$
\Gamma(k-\alpha) / \Gamma(k)<K k^{-\alpha} \quad \text { for } \quad k=1,2, \cdots,
$$

which follows immediately from the fact that $\lim _{k \rightarrow \infty} \Gamma(k+1-$ $\alpha) / \Gamma(k) k^{1-\alpha}=1$ (see [4], p. 195). Using (3.2) we have that

$$
\begin{aligned}
& (t / n)^{1-\alpha} \sum_{k=1}^{n} \Gamma(k-\alpha) e^{-\imath \beta k / n} / \Gamma(k) \\
& \quad \leqq t^{1-\alpha} K \sum_{k=1}^{n}\left(e^{-\imath \beta k / n}(k / n)^{-\alpha}\right)(1 / n) .
\end{aligned}
$$

Since $\sum_{k=1}^{n}\left(e^{-i \beta k / n}(k / n)^{-\alpha}\right)(1 / n)$ is a lower approximating sum to the integral $\int_{0}^{1} e^{-i \beta x} x^{-\alpha} d x$, (3.1) follows immediately using (1.11).

Lemma 3.2. Let (1.3) hold and let $\alpha \in(0,1)$. If $x \in X, t \geqq 0$, and $n$ is a positive integer,

$$
\begin{array}{r}
\left\|(I+t / n A)^{-k} x\right\|_{\alpha} \leqq(n / t)^{\alpha} C \Gamma(k-\alpha)(1-\omega t / n)^{\alpha-k}\|x\| / \Gamma(k) \\
\text { for } k=1, \cdots, n .
\end{array}
$$

Proof. We will use the formula

$$
(\lambda I+A)^{-k}=\int_{0}^{\infty} e^{-\lambda s} s^{k-1} T(s) x d s /(k-1) !
$$


for $\lambda>\omega, k=1,2, \cdots$ (see [2], p. 623). From (3.4) we obtain

$$
\begin{aligned}
& \left\|(I+t / n A)^{-k}\right\|_{\alpha} \\
& =\left\|(n / t)^{k} \int_{0}^{\infty} e^{-n s / t} s^{k-1} A^{\alpha} T(s) x d s /(k-1) !\right\| \\
& \quad \leqq(n / t)^{k} \int_{0}^{\infty} C e^{(\omega-n / t) s} s^{k-1-\alpha}\|x\| d s / \Gamma(k)
\end{aligned}
$$

and (3.3) follows immediately using (1.11).

Lemma 3.3. Let (1.3) and (1.7) hold. If $t>0$ and $n$ is a positive integer sufficiently large, then

(3.5) $(I+t / n F)^{-1}$ exists as an everywhere defined mapping from $\left(D\left(A^{\alpha}\right)\right.$ into $D\left(A^{\alpha}\right)$;

(3.6) for $x \in D\left(A^{\alpha}\right)$ and $\left.j=1,2, \cdots, n,(I+t / n) F\right)^{-j} x=(I+t / n A)^{-j} x$

$$
-(t / n) \sum_{k=1}^{j}(I+t / n A)^{-k} B(I+t / n F)^{-(j-k+1)} x .
$$

Proof. Let $x \in D\left(A^{\alpha}\right)$, let $t>0$, let $n$ be a positive integer, and define $G: D\left(A^{\alpha}\right)$ to $D\left(A^{\alpha}\right)$ by

$$
G(y)=(I+t / n A)^{-1}(x-t / n B y), \quad y \in D\left(A^{\alpha}\right)
$$

(note that $G$ maps into $D(A) \subset D\left(A^{\alpha}\right)$, see [3], p. 159). From (3.3) and (1.11) we obtain for $y_{1}, y_{2} \in D\left(A^{\alpha}\right)$

$$
\left\|G\left(y_{1}\right)-G\left(y_{2}\right)\right\|_{\alpha} \leqq C \Gamma(1-\alpha)(1-\omega t / n)^{\alpha-1}(t / n)^{1-\alpha} L\left\|y_{1}-y_{2}\right\|_{\alpha} .
$$

For $n$ sufficiently large $G$ is a contraction from $D\left(A^{\alpha}\right)$ to $D\left(A^{\alpha}\right)$ and has a unique fixed point $y$ which satisfies $(I+t / n(A+B)) y=x$. (3.5) follows immediately and a simple induction argument proves (3.6).

Remark 3.1. By virtue of (3.3), (3.6), and Corollary 2, p. 241 of [9] we have that $D(F)$ is dense in $D\left(A^{\alpha}\right)$, since

$$
\begin{aligned}
\left\|(I+t / n F)^{-1} x-x\right\|_{\alpha} \leqq & \left\|(I+t / n A)^{-1} A^{\alpha} x-A^{\alpha} x\right\| \\
& +(t / n)\left(\left\|A^{\alpha}(I+t / n A)^{-1}\left(B(I+t / n F)^{-1} x-B x\right)\right\|\right. \\
& \left.+\left\|A^{\alpha}(I+t / n A)^{-1} B x\right\|\right) .
\end{aligned}
$$

Lemma 3.4. Let (1.3) and (1.7) hold, let $x \in D\left(A^{\alpha}\right), t_{0}>0$, and $\epsilon>0$. There exists a positive integer $N$ such that if $n \geqq N, 1 \leqq j \leqq n$, and $0 \leqq t \leqq t_{0}$, then 


$$
\begin{array}{r}
\|(t / n) \sum_{k=1}^{l}(I+t / n A)^{-k} B U(t(j-k+1) / n) x \\
-\int_{0}^{t / n} T(s) B U((t j / n) \cdot s) x d s \|_{\alpha}<\epsilon .
\end{array}
$$

Proof. Let $\left\{y_{1}, \cdots, y_{m}\right\}$ be a finite set in $\left(D\left(A^{\alpha}\right)\right.$ such that if $0 \leqq s \leqq t_{0}$, then there exists some integer $i \in[1, m]$ such that $\left\|B U(s) x-y_{i}\right\|<\epsilon$ (here we use the fact that $D\left(A^{\alpha}\right)$ is dense in $X$ and $B A^{-\alpha} A^{\alpha} U(s) x$ is continuous in $s$ from $\left[0, t_{0}\right]$ to $X$ and hence has compact range). Choose $N$ such that if $n \geqq N$, then both of the following conditions hold:

$\left\|(t / n) \sum_{k=1}^{j}\left((I+t / n A)^{-k}-T(t k / n)\right) y_{1}\right\|_{\alpha}<\epsilon \quad$ for $\quad$ all $\quad t \in\left[0, t_{0}\right]$, $j=1, \cdots, n$, and $i=1, \cdots, m$ (here we use the fact that for $z \in X$, $\lim _{n \rightarrow \infty}\left\|(I+s / n A)^{-n} z-T(s) z\right\|=0$ uniformly for $0 \leqq s \leqq t_{0}$, see [6], p. 481);

$$
\begin{gathered}
\left\|(t / n) \sum_{k=1}^{L} T(t k / n) z_{k, j, n}-\int_{0}^{t / n} T(s) B U((t j / n)-s) x d s\right\|_{\alpha}<\epsilon \text { for all } \\
t \in\left[0, t_{0}\right] \text { and } j=1, \cdots, n \text {, where } z_{k,, n}=B U(t(j-k+1) / n) x .
\end{gathered}
$$

Using Lemma 3.1 we can find a constant $K_{1}$ such that for all $n=1,2, \cdots$, and $0 \leqq t \leqq t_{0}$

$$
(t / n)^{1-\alpha} \sum_{k=1}^{n} C \Gamma(k-\alpha)(1-\omega t / n)^{\alpha-k} / \Gamma(k) \leqq K_{1} .
$$

Also, there exists a constant $K_{2}$ such that for all $n=1,2, \cdots$, and $0 \leqq t \leqq t_{0}$

$$
(t / n) \sum_{k=1}^{n} C e^{\omega k t / n}(t k / n)^{-\alpha}<K_{2} .
$$

Now let $n \geqq N$, let $0 \leqq t \leqq t_{0}$, let $1 \leqq j \leqq n$, and for each $k=1, \cdots, j$ choose an integer $i(k) \in[1, m]$ such that $\left\|z_{k, l, n}-y_{i(k)}\right\|<\epsilon$. Then, using Lemma 3.2 and (3.8) we have

$$
\begin{aligned}
& \left\|(t / n) \sum_{k=1}^{1}\left((I+t / n A)^{-k}-T(t k / n)\right) z_{k, j, n}\right\|_{\alpha} \\
& \leqq(t / n)^{1-\alpha} \sum_{k=1}^{j}\left(C \Gamma(k-\alpha)(1-\omega t / n)^{\alpha-k} / \Gamma(k)\right)\left\|z_{k,, n}-y_{t(k)}\right\| \\
& +\left\|(t / n) \sum_{k=1}^{1}\left((I+t / n A)^{-k}-T(t k / n)\right) y_{l(k)}\right\|_{\alpha} \\
& +(t / n) \sum_{k=1}^{n} C e^{t k \omega / n}(t k / n)^{-\alpha}\left\|y_{i(k)}-z_{k, j, n}\right\|<K_{1} \epsilon+\epsilon+K_{2} \epsilon .
\end{aligned}
$$


Then, (3.7) follows immediately from (3.9) and (3.10).

Theorem. Let (1.3) and (1.7) hold and let $x \in D\left(A^{\alpha}\right)$. Then, uniformly in bounded intervals of $t$

$$
\lim _{n \rightarrow \infty}\left\|(I+t / n F)^{-n} x-U(t) x\right\|_{\alpha}=0 .
$$

Proof. Let $t_{0}>0$. Let $K$ be a constant as in Lemma 3.1 and observe that

$$
(1-\omega t / n)^{\alpha-k} \leqq 1 \quad \text { for } \quad n \geqq 1, \quad k=1, \cdots, n, 0 \leqq t \leqq t_{0}
$$

Let $\beta>0$ such that $C L K \Gamma(1-\alpha) \beta^{\alpha-1}<1 / 4$. Let $\epsilon>0$ and choose $N$ such that if $n \geqq N$ then (3.7) holds, as well as the following:

$$
\left\|(I+t / n A)^{-j} x-T(t j / n) x\right\|_{\alpha}<\epsilon \text { for } j=1, \cdots, n, 0 \leqq t \leqq t_{0} ;
$$

(3.14) $e^{t \beta / n}<2$ for $0 \leqq t \leqq t_{0}$. Now fix $n \geqq N, 0<t \leqq t_{0}$, and define

$$
w_{j}=\left\|(I+t / n F)^{-j} x-U(t j / n) x\right\|_{\alpha}, j=1, \cdots, n, S_{n}(t)=\sup _{1 \leqq j \leqq n} e^{-t \beta j / n} w_{j} .
$$

Using (3.12), (3.13), (3.14), and Lemmas 3.1, 3.2, 3.3, and 3.4 we have for $j=1, \cdots, n$

$$
\begin{aligned}
w_{j}= & \|(I+t / n A)^{-j} x+(t / n) \sum_{k=1}^{j}(I+t / n A)^{-k} B(I+t / n F)^{-(j-k+1)} x \\
& -T(t j / n) x-\int_{0}^{t / n} T(s) B U(t j / n-s) x d s \|_{\alpha} \\
\leqq & \left\|(I+t / n A)^{-j} x-T(t j / n) x\right\|_{\alpha} \\
& +\|(t / n) \sum_{k=1}^{j}(I+t / n A)^{-k}\left(B(I+t / n F)^{-(j-k+1)} x\right. \\
& \quad-B U(t(j-k+1) / n) x \|_{\alpha} \\
& +\|(t / n) \sum_{k=1}^{j}(I+t / n A)^{-k} B U(t(j-k+1) / n) x \\
& \quad-\int_{0}^{t j / n} T(s) B U((t j / n)-s) x d s \|_{\alpha} \\
< & +(t / n)^{1-\alpha} \sum_{k=1}^{j}(C \Gamma(k-\alpha) / \Gamma(k)) L w_{j-k+1}+\epsilon .
\end{aligned}
$$

From (3.15) we obtain for $j=1, \cdots, n$ 
$e^{-t \beta j / n} w_{J}$

$$
\begin{aligned}
& <2 \epsilon+(t / n)^{1-\alpha} \sum_{k=1}^{j}\left(C L \Gamma(k-\alpha) e^{-t \beta k / n} / \Gamma(k)\right)\left(e^{t \beta / n}\right)\left(e^{-t \beta(j-k+1) / n} w_{\jmath-k+1}\right) \\
& <2 \epsilon+2 C L K \Gamma(1-\alpha) \beta^{\alpha-1} S_{n}(t) \\
& <2 \epsilon+S_{n}(t) / 2
\end{aligned}
$$

Then, (3.16) implies $S_{n}(t)<2 \epsilon+S_{n}(t) / 2$ or, equivalently, $S_{n}(t)<$ $4 \epsilon$. (3.11) follows immediately and the proof is complete.

In conclusion we remark that our methods can be used to treat the existence and exponential representation of local solutions to (1.1) in the case that $B$ satisfies a local Lipschitz continuity condition from $D\left(A^{\alpha}\right)$ to $X$. Also, our methods can be used in the numerical study of (1.1), a program which we will carry out elsewhere.

\section{REFERENCES}

1. M. G. Crandall and T. M. Liggett, Generation of semigroups of nonlinear transformations on general Banach spaces, Amer. J. Math., 93 (1971), 265-298.

2. N. Dunford́ and J. Schwartz, Linear Operators, Part I., Interscience, New York, 1957.

3. A. Friednan, Partial Differential Equations, Holt, Rinehart, and Winston, New York, 1969.

4. J. Gerretsen and G. Sansone, Lectures on the Theory of Functions of a Complex Variable, P. Noordhoff, Groningen, 1960.

5. D. Henry, Geometric Theory of Nonlinear Parabolic Equations, to appear.

6. T. Kato, Perturbation Theory for Linear operators, Springer-Verlag, New York, 1966.

7. K. Maruo, Integral equation associated with some non-linear evolution equation, J. Math. Soc. Japan, 26 (1974), 433-439.

8. G. Webb, Continuous nonlinear perturbations of linear accretive operators in Banach spaces, J. Functional Analysis, 10 (1972), 191-203.

9. K. Yosida, Functional Analysis, Springer-Verlag, New York, 1968.

Received January 28, 1976

VANDERBILT UNIVERSITY

NASHVILLE, TN 37235 




\section{Pacific Journal of Mathematics \\ Vol. 70, No. $1 \quad$ September, 1977}

William H. Barker, Noether's theorem for plane domains with hyperelliptic

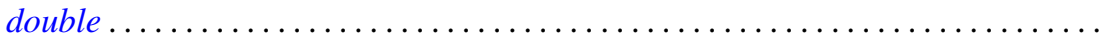

Michael James Beeson, Non-continuous dependence of surfaces of least area on the boundary curve ...................................... 11

Horst Behncke, Functions acting in weighted Orlicz algebras . . . . . . . . . . . . 19

Howard Edwin Bell, A commutativity study for periodic rings . . . . . . . . . . . 29

Peter Botta and Stephen J. Pierce, The preservers of any orthogonal group ....... 37

Douglas S. Bridges, The constructive Radon-Nikodým theorem ............. 51

James Dennis Brom, The theory of almost periodic functions in constructive

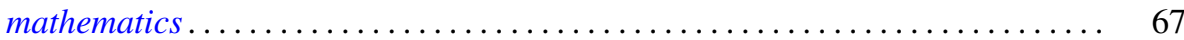

N. Burgoyne and C. Williamson, Semi-simple classes in Chevalley type groups ....

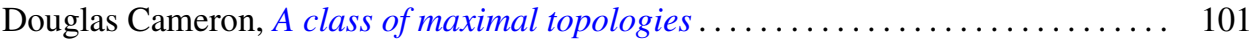

L. Carlitz, Enumeration of doubly up-down permutations . . . . . . . . . . . . . . 105

Paul Robert Chernoff, The quantum n-body problem and a theorem of

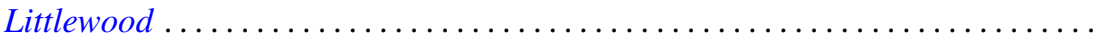

Jo-Ann Deborah Cohen, Locally bounded topologies on $F(X) \ldots \ldots \ldots \ldots \ldots \ldots$

Heinz Otto Cordes and Robert Colman McOwen, Remarks on singular elliptic

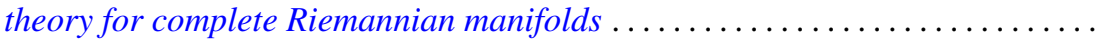

Micheal Neal Dyer, Correction to: "Rational homology and Whitehead

products"

Robert Fernholz, Factorization of Radonifying transformations

Lawrence Arthur Fialkow, A note on quasisimilarity. II ...... . .

Harvey Charles Greenwald, Lipschitz spaces of distributions on the surface of unit

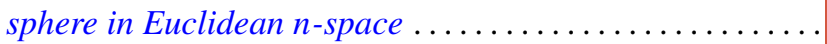

Albrecht Irle, On the measurability of conditional expectations

Tom (Roy Thomas Jr.) Jacob, Matrix transformations involving simple sequence

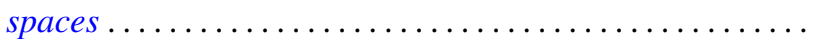

A. Katsaras, Continuous linear maps positive on increasing continuous

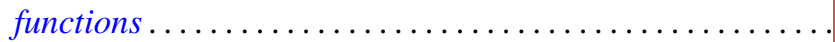

Kenneth Kunen and Judith Roitman, Attaining the spread at cardinals of cofinality

Lawrence Louis Larmore and Robert David Rigdon, Enumerating normal bundles

of immersions and embeddings of projective spaces ...... . .

Ch. G. Philos and V. A. Staïkos, Asymptotic properties of nonoscillatory solutions of differential equations with deviating argument .

Peter Michael Rosenthal and Ahmed Ramzy Sourour, On operator algebras containing cyclic Boolean algebras...

Polychronis Strantzalos, Strikt fast gleichgradig-stetige und eigentliche

Aktionen ...

Glenn Francis Webb, Exponential representation of solutions to an abstract

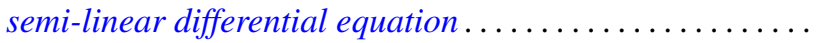

